
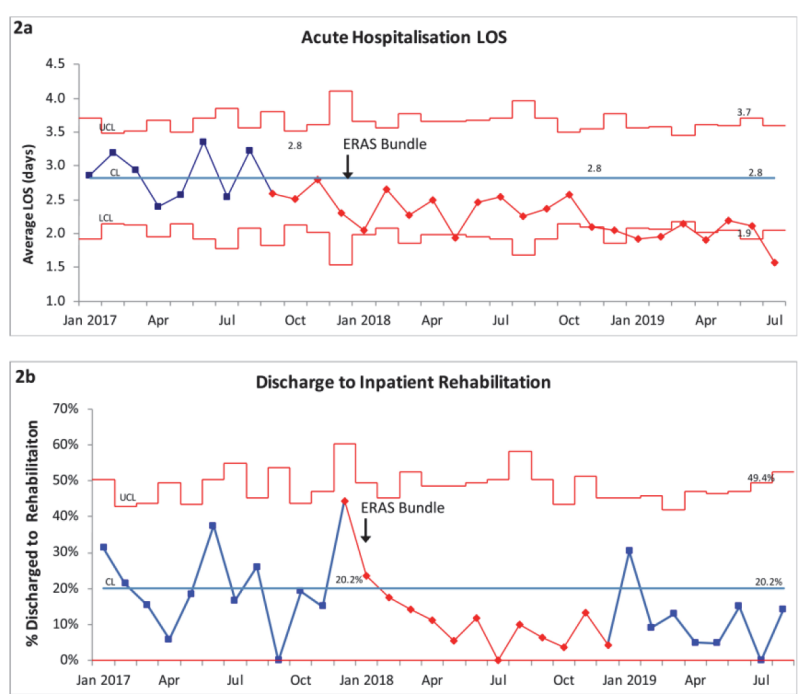

Abstract 5 Figure 3 Statistical process control chart for acute hospitalization length of stay

Abstract 5 Table 1 Characteristics of patients undergoing TKA

\begin{tabular}{lccccc}
\hline & \multicolumn{2}{c}{ Pre-ERAS Group } & \multicolumn{2}{c}{ ERAS bundle } & $p$ value \\
\hline Number of patients & \multicolumn{2}{c}{232} & \multicolumn{3}{c}{383} \\
\hline Age, year (mean, & 66.1 & $(10.1)$ & 66.5 & $(9.9)$ & 0.466 \\
(SD)) & & & & & \\
Female, $n$ & 148 & $(63.8 \%)$ & 228 & $(59.5 \%)$ & 0.334 \\
ASA classification & & & & & 0.090 \\
ASA I & 4 & $(1.7 \%)$ & 5 & $(1.3 \%)$ & \\
ASA II & 97 & $(41.8 \%)$ & 123 & $(32.1 \%)$ & \\
ASA III & 126 & $(54.4 \%)$ & 243 & $(63.4 \%)$ & \\
ASA IV & 5 & $(2.2 \%)$ & 12 & $(3.1 \%)$ & \\
BMI, kg/m ${ }^{2}$ (mean, & 31.0 & $(7.0)$ & 32.45 & $(7.83)$ & 0.023 \\
(SD)) & & & & & \\
Neuraxial & 199 & $(85.8 \%)$ & 329 & $(85.9 \%)$ & 1.000 \\
anaesthetic & & & & & \\
\end{tabular}

TKA = total knee arthroplasty, ERAS = enhanced recovery after surgery, ASA = American Society of Anesthesiologists, BMI = body mass index

Abstract 5 Table 2 Interrupted time series analysis (ITS) used to model monthly LOS and percent discharged to inpatient rehabilitation

\begin{tabular}{lcccc}
\hline Outcome & $\begin{array}{c}\text { Final Month of } \\
\text { Pre- } \\
\text { Intervention }\end{array}$ & $\begin{array}{c}\text { Final Month of } \\
\text { Post- } \\
\text { Intervention }\end{array}$ & Difference & $\begin{array}{c}\text { Wald } p \\
\text { value }\end{array}$ \\
\hline LOS (days) & 2.60 & 1.81 & -0.79 & $<0.001$ \\
& {$[2.30,2.90]$} & {$[1.59,2.03]$} & {$[-1.16,-$} & \\
& & & $0.42]$ & \\
LOS < 2 Days & $18.3 \%$ & $69.3 \%$ & $50.9 \%$ & $<0.001$ \\
Discharge to & {$[9.0,27.8]$} & {$[62.4,76.1]$} & {$[39.3,62.6]$} & \\
Rehabilitation & $19.9 \%$ & $8.2 \%$ & $-11.7 \%$ & 0.045 \\
\hline
\end{tabular}

LOS = length of stay
Abstract 5 Table 3 Outcome, process, and balance measures

\begin{tabular}{lcccccc}
\hline & Pre ERAS Bundle & ERAS Bundle & P value \\
\hline Number of patients & 282 & & 383 & & \\
\hline Hospitalization LOS, days (mean, (SD)) & 2.82 & $(1.25)$ & 2.13 & $(1.09)$ & $<0.001$ \\
Inpatient rehabilitation & 47 & $(20.2 \%)$ & 41 & $(10.7 \%)$ & 0.002 \\
24-hour oral morphine, mg (mean, (SD)) & 59.7 & $(76.41)$ & 38.05 & $(52.42)$ & $<0.001$ \\
Maximum VRS pain score first 24 hours & & & & & $<0.001$ \\
No pain & 8 & $(3.6 \%)$ & 47 & $(12.7 \%)$ & \\
Mild & 54 & $(24.2 \%)$ & 148 & $(40.1 \%)$ & \\
Moderate & 97 & $(43.5 \%)$ & 113 & $(30.6 \%)$ & \\
Severe & 64 & $(28.7 \%)$ & 61 & $(16.5 \%)$ & \\
Post-operative Nausea and Vomiting & 120 & $(51.7 \%)$ & 140 & $(36.6 \%)$ & $<0.001$ \\
Adductor Canal Block & 35 & $(15 \%)$ & 250 & $(65 \%)$ & $<0.001$ \\
IV dexamethasone & 49 & $(21 \%)$ & 244 & $(64 \%)$ & $<0.001$ \\
Foley Catheterization & & & & & \\
Pre-operative & 221 & $(95.3 \%)$ & 61 & $(15.9 \%)$ & $<0.001$ \\
Post-operative & 4 & $(1.7 \%)$ & 78 & $(20.4 \%)$ & $<0.001$ \\
Post discharge 30-day ED visits & 30 & $(12.9 \%)$ & 28 & $(7.3 \%)$ & 0.030 \\
\hline
\end{tabular}

ERAS = enhanced recovery after surgery, LOS = length of stay, VRS = verbal rating scale IV = intravenous, ED = emergency department

$(p<0.001)$. The percentage of patients experiencing moderateto-severe pain and postoperative nausea and vomiting within the first 24 -hours decreased by $25 \%$ and $15 \%$, respectively $(\mathrm{p}<0.001)$. 30-day emergency department visits following discharge decreased by 5\% $(\mathrm{p}=0.030)$ (table 3 , figures 2 and 3 ). Conclusions Significant improvements in the recovery of patients after TKA were achieved by performing a RCA and implementing a multi-disciplinary, patient-centered ERAS bundle.

\section{REDUCING UNNECESSARY PATIENT ISOLATION ON GENERAL MEDICINE UNITS}

${ }^{1}$ Joseph Carson, ${ }^{2}$ Mary-Margaret Taabazuing, ${ }^{2}$ Cody Sider, ${ }^{2}$ Michael Payne, ${ }^{2}$ Yassmin Behzadian, ${ }^{3}$ Alice Newman, ${ }^{3}$ Elaine Hunter Gutierrez, ${ }^{3}$ Linda Elliot, ${ }^{3}$ Brittany Devoe. 'Western University; London Rheumatology, Canada; ${ }^{2}$ Western University; London Health Sciences Centre, Canada; ${ }^{3}$ London Health Sciences Centre, Canada

\subsection{6/bmjoq-2020-IHI.6}

Background Droplet+contact (DC) precautions are used to prevent the spread of acute respiratory infections. Clinicians at London Health Sciences Centre, an academic tertiary care organization in Ontario, Canada, have reported that many patients remain isolated longer than necessary. Research suggests that prolonged isolation may negatively impact patient outcomes, experience, and costs.

Objectives Reduce unnecessary DC precautions on general medicine units by 30\% by March 31, 2020.

\section{ISOLATION PROJECT: APPENDIX}

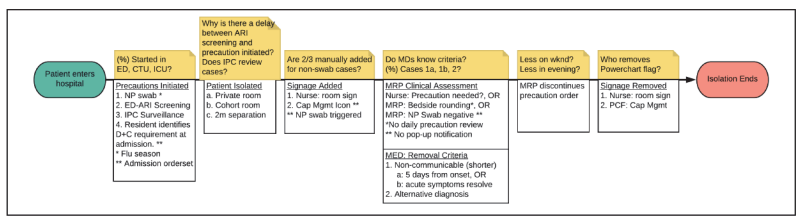

Abstract 6 Figure 1 Current state process map 
Methods Our multi-disciplinary team designed this project using the Model for Improvement. We identified barriers to precaution removal through surveys, chart reviews, process mapping (figure 1), and fishbone diagramming (figure 2). Our change drivers focussed on motivation, precaution identification, reassessment cues, and standardized decision-making (figure 3). In a series of PDSA cycles, we tested and

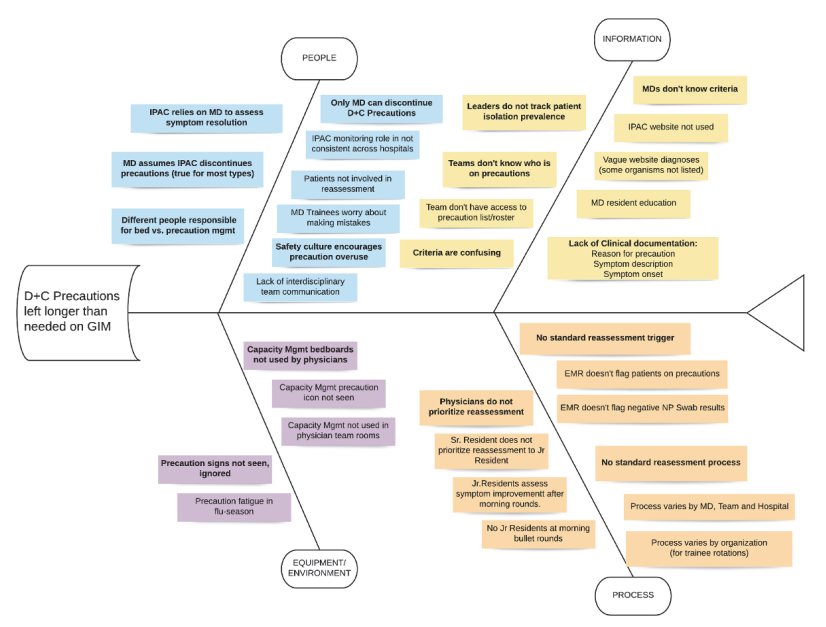

Abstract 6 Figure 2 Fishbone barriers to removal

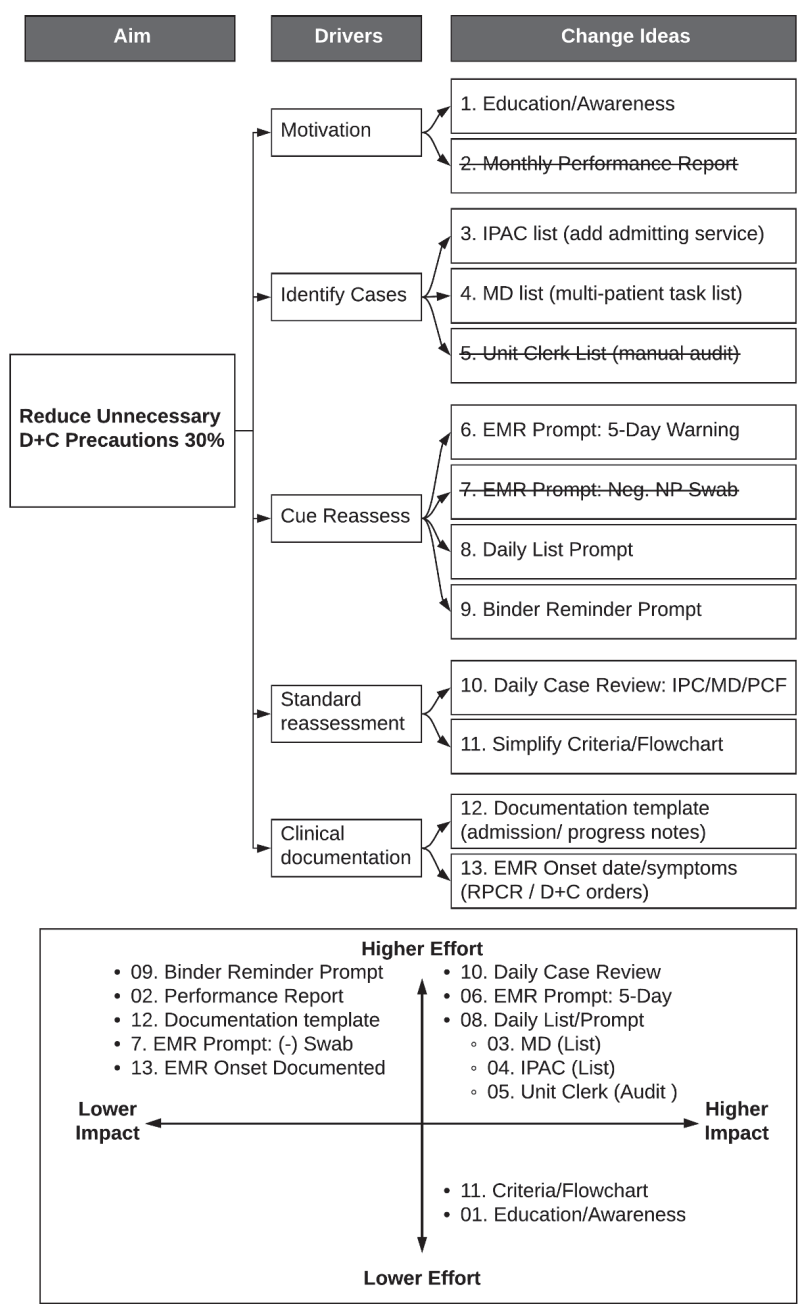

Abstract 6 Figure 3 Driver diagram and priority matrix
When to discontinue Droplet+Contact Precautions

(+) RSV A/B or Influenza A/B: Choose the longer option

- Afebrile for 48 hours

- 5 days from admission or symptom onset **

(-) RSV A/B or Influenza A/B: Choose the shorter option

- Acute symptoms resolved

- Alternative diagnosis confirmed

- 5 days from admission or symptom onset

** 7 days for transplant recipients, critical care patients, pediatric patients, and oncology unit patients

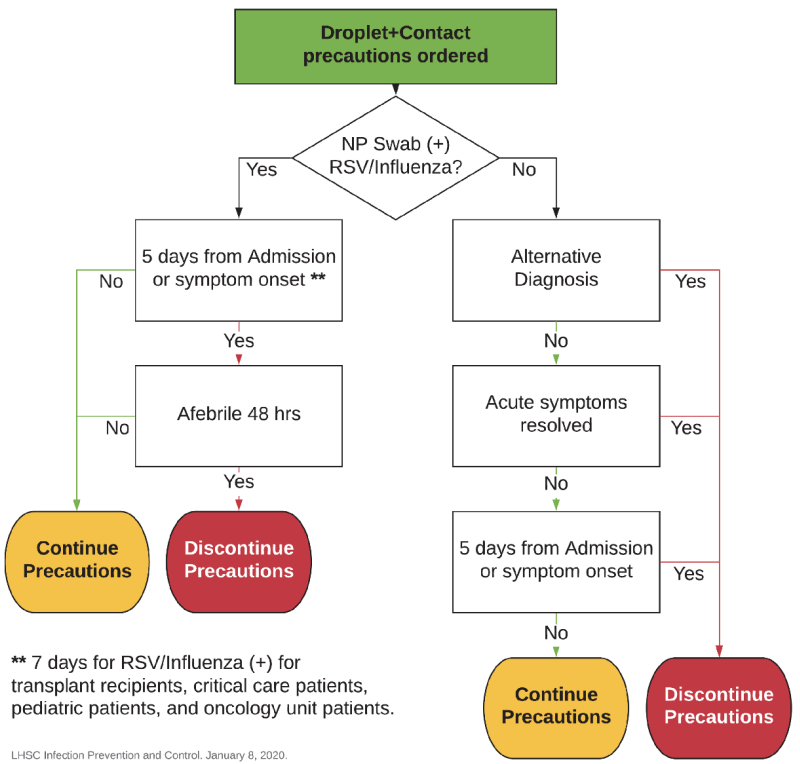

Abstract 6 Figure 4 Criteria and decision support tool

implemented new discontinuation criteria and a decision-support tool across two hospitals (figure 4). Outcomes measures were: (1) \% unnecessary DC precautions, collected by weekly physician audits, and (2) DC precautions lasting $>5$ days, collected from electronic medical records. Our process measures were: (1) user test fidelity, and (2) physician awareness. Our balance measure was physician satisfaction with new criteria. Statistical analysis was performed using Student's t-test, run charts, and process control charts (QI Macros, IHI Rules).

Results We completed eight appropriateness audits $(\mathrm{n}=212$ patients) at two hospitals between December 2019 - March 2020. During user testing, eight physicians applied the new criteria and decision-support tool to five mock cases at $92 \%$ (37/40) fidelity. After implementing changes, mean precaution appropriateness increased from $30 \%(24 / 80)$ to $64 \%(85 / 132)$, $(\mathrm{p}<0.001)$. Out of 35 physicians surveyed, $22(63 \%)$ were aware of new criteria; of those, 19 (86\%) found the new criteria useful. However, there was no special-cause variation in DC precautions $>5$ days.

Conclusions Discontinuing prolonged DC precautions is important to conserve vital resources, especially during the COVID19 pandemic. We reduced these incidents by implementing standard discontinuation criteria and a decision support tool. Our next step is to adapt these tools to standardize precaution removal for COVID-19 patients. 